\title{
SOME DIFFERENTIAL CHARACTERISTICS OF CEREBRAL MOTOR DEFECTS IN INFANCY
}

\author{
BY \\ EIRENE COLLIS \\ From the Cerebral Palsy Research Clinic and Parents' Advice Clinic, Queen Mary's Hospital for Children, Carshalton
}

(RECEIVED FOR PUBLICATION DECEMBER 7, 1953)

During the 13 years in which $I$ have been studying the characteristics of physical activity of babies and young children with motor defects of cerebral origin I have again and again been confronted with diagnoses which describe presenting signs simply as 'pyramidal' or 'extrapyramidal'. With the greatest deference I would urge the importance of clinical analysis of available function in each affected child. Physical activity-the expression of interrelated activity of neural structures-appears as a single thing, a living mosaic showing cerebral function both mental and motor. Physical signs are signs of function; where the brain is incomplete they show function which by its abnormality reveals the absence of neural activity indispensable to normal function, that is, they show the presence of a lesion or lesions. A diagnosis of, for instance, 'pyramidal defect' is thus based on the location, by deduction, of inactive cells. Yet the defect is manifest through the behaviour of active cells of infinitely complex function-motor and mental. Hence, the terms pyramidal or extrapyramidal signs are not by themselves adequate to describe the nature of that remaining function upon which the child's potentialities and the planning of treatment depend.

The presentation of such elementary data is excusable on the grounds that little is known of the growth of function which lacks normal components, and it is only now becoming recognized that signs in the physical activity of a child with an incomplete brain show change as that brain matures. By comparison with the physical activity seen in normal children of the same age the clinician may assess the function, both mental and motor, remaining to a child with a non-progressive lesion of the brain and plan for its fullest exploitation. In this paper I am confining myself to a description of some of the less striking characteristics of physical activity in growing children with abnormalities of motor function from which, I believe, the nature of their residual motor function may be deduced. (I have described elsewhere (Collis, 1953) some characteristics of mental activity to be seen in the physical behaviour of infants.) The achievements of motordeficient children rest not only on their mental capacity but also on the kind of physical handling they receive during the stages of 'plasticity' of the nervous system. For this reason it is important that their remaining equipment-mental, motor and special sense-should be assessed as early as possible in life and help provided for those of sufficient mental capacity.

\section{Diagnosis of Motor Abnormality in Infancy}

It is not yet universally accepted that diagnosis of motor defect in infancy is important. It is important because inappropriate handling of affected babies augments their motor abnormality, and handling which is based on the assumption that an affected baby is normal inevitably is inappropriate. It is not always accepted that differential diagnosis is important, yet it is important because treatment must be appropriate to the type of motor abnormality as well as to the child's mental status. Nor is it generally recognized that diagnosis of brain abnormality in infancy is possible where there are no gross signs. We find, however, that a history of defective breathing and sucking throughout infancy is commonly given in cases of motor abnormality and that most of these children are brought to the doctor in babyhood because the parents suspect that all is not well. This means that signs of neural abnormality are indeed present in babyhood though they are not the signs in the adult with neurological motor defect. Neurological signs must be specially looked for in infancy to prevent delay in instructing parents in the techniques of handling their motordeficient babies.

For practical purposes, cerebral function in the infant may be deduced from observation of the infantile reflexes; these are fragments of the primary activity of the newborn baby and may be seen as he 
is handled. The human brain is concerned, however, with thought as well as with movement, and in so-called voluntary movement, which includes speech, thought finds expression through the reflex motor apparatus. The change from infantile to mature activity happens in a gradual way and is most easily seen by observing modifications of the infantile reflexes which can be used as guides for assessing the normality or abnormality of developing mental activity.

Brain abnormality may affect mental processes and leave reflex motor activity intact. Modification of the infantile reflexes shows abnormality in such cases; in fact many workers have noted imperfect modification of infantile characteristics in mentally defective subjects; the grasping reflex, for instance, may persist in a relatively unmodified form up to a late age. At all ages mentally defective children show abnormality in their use of movement. On the other hand, brain abnormality may directly disorder reflex motor activity itself so that the infantile reflexes, the sucking reflex, for instance, are impaired and the abnormality is in movement itself and hence developing voluntary activity is distorted. Widespread abnormality of brain function affecting both the mental and motor aspects of physical activity is shown by a deficient use of movement which itself is abnormal. Though in the individual case movement itself, or its expressive use, or both parts of physical activity at once may be impaired by loss of brain cells, at examination the clinician is confronted with the two aspects simultaneouslyphysical activity. In order to diagnose abnormality and recognize normality in either aspect he must analyse this physical activity and distinguish between the two parts.

\section{Alterations of Muscle Tone}

That the normal young infant shows hypotonicity is well known, yet babies' limbs resist reflexly direct attempts to alter their flexed attitudes; both the hypotonicity and the resistance diminish as development proceeds. These and other changes express the evolution of intact brain activity; similarly, with the deficient brain, evolution of residual brain activity is expressed by changes in physical activity. Reflex motor activity is gradually modified during the stages of infant development and throughout life it serves to express thought, so even where this reflex function is defective motor signs change with mental development and movement is modified by thought. In mentally defective babies physical expression continues to be characteristically infantile; on the other hand, where developing mental activity can find only distorted expression because of motor insufficiency, there are characteristic signs of weakness and inadequate progress. Thus in cases of motor abnormality it is essential to distinguish modifications of movement which are related to mental activity from morbid signs. Though this distinction may not be easy, when signs are viewed as a whole and due weight is given to effects of any coexistent defect such as deafness, and if the malleability of nervous function in the young child is duly remembered, it is usually possible to estimate correctly available function, both motor and mental, in any given case.

While in children with the type of motor insufficiency characterized by ataxia there is no stiffness, in other types, though hypotonus remains as a sign, stiffening takes place as the child gets older. (Stiffness or opisthotonus in the very young baby occur among other signs of gross cerebral defect and present little diagnostic difficulty.) Where stiffening gradually develops as the infant gets older, this may be neuro-pathological in origin or may occur as force is exerted by or emotion aroused in the affected child. There are thus two possible sources of stiffening - the motor apparatus and the mental apparatus. In the child with an intact central nervous system stiffening of effort or emotion, even when widespread and associated with grimacing, is readily correctly interpreted by the observer, but in the motor-deficient child such stiffening may easily be mistaken for added dysfunction and, as such, attributed to the presence of a further lesion of the motor apparatus. Signs of mental activity, including this normal characteristic of stiffening, are not discernible in the very young baby and are absent or greatly diminished at physiological rest, for instance in sleep, in the older subject. When effects of mental activity-normal and abnormal-are subtracted from deficient physical activity, remaining characteristics indicate the nature of the motor function available to the child, and it is on these characteristics, which may or may not include stiffness, that differential diagnosis of the type of palsy should be based.

We have been able to distinguish only two types of stiffening of neuropathological origin in cases of infantile cerebral palsy; these we call spasticity and rigidity. Stiffness of either type varies in degree from case to case, but the reactions characterizing each type remain constant. While the type of stiffness we describe as rigidity may be present in the very young baby, the type we describe as spasticity develops relatively slowly. A difference in the early histories of children showing these abnormalities is usual. Rigidity is often a sequel of prolonged jaundice or of white asphyxia, while spasticity may develop in 
a child who suffered birth trauma or in one who was normally born. Mental insufficiency (unless associated with specific physical abnormality such as microcephaly) cannot be diagnosed in the newborn child, but when gross signs of cerebral abnormality are present in early infancy the prognosis is poor; if a child has fits a guarded prognosis should always by given. In cases of rigidity a history of early signs is commonly given, and mental defect is, in our experience, invariable. In cases of spasticity and in cases where stiffness of non-pathological origin develops, a history of early signs may not be given; we think that in such cases less prominent signs have been missed. Usually, affected children show abnormal hypotonus and lack of normal advance throughout infancy, but in the absence of gross signs they are regarded as normal until they develop deformities.

\section{Spasticity}

The neuromotor abnormality we describe as infantile generalized spasticity can usually be differentiated from other types of motor defect before it attains its recognized clinical form and before the usual clinical tests for pyramidal defect are of value. Though spastic stiffness develops relatively slowly, two early signs indicate the presence of the neurological defect underlying its development. Where both these signs are present in a child up to 2 or 3 years of age the development of spasticity should be expected. First, with the child supine, the ankles are firmly grasped, the thighs rotated inward and sharply abducted (Fig. 1) resistance is abruptly encountered. Second, with the child prone, with his pelvis held in contact with the surface on which he is lying, one of his legs is passively flexed at the knee and the sole of that foot stroked firmly with the examiner's thumb nail (Figs. 4 and 5); the hip on the same side flexes. (Each reaction may be obtained repeatedly, and it should be noted that the second reaction differs from the well-known infantile 'withdrawal' which may persist in mentally deficient children whether or not motor dysfunction is also present.)

In infantile generalized spasticity postures of the limbs and body of the older infant show little change from those seen in theyoung baby; in most cases signs of mental defect are found. Though the young affected child is noticeably inactive and hypotonic, the Moro reflex is readily evoked; the sharp contrast between his slow and laboured voluntary activity and this hyperreflexia is often mistakenly regarded as evidence of abnormal 'nervousness' and fear. It is not fear; even the normal baby shows this sign at the time of birth before the emotion of fear can safely be attributed to him. Later on fear and 'the body jerk' may become related, but we cannot assume the presence of fear in the child with motor dysfunction who exhibits this hyperreflexia as a feature of his condition. Such a child cannot develop normal security of posture and is easily thrown off physical balance; it seems to me that physical insecurity, spastic stiffness, and inadequate modification of infantile reflexia sufficiently account for the supposed 'highly nervous' state of many affected children.

With the development of voluntary activity the deficient reflex motor apparatus produces that stiffening which indicates the nature of the abnormality; this spasticity is subject to modification and decreases as the child becomes more adept and speedy in the purposeful use of his limbs. In older children the gross clinical picture of Little's disease is seen only where there is severe mental defect, but in many children there is marked spasticity only in the legs; this develops during the period of evolution of use of the upper limbs when movements of the legs are still largely reflex. Voluntary activity of the baby's arms sets in train reflex leg movements; the baby drags his lower limbs when trying to crawl forward by using his forearms for support and so activates his abnormal reflex leg patterns; where these are subsequently reinforced by premature attempts to assist the development of walking, the impaired reflex apparatus has full play to establish grossly distorted lower limb activity. Such children require skilled assistance in all early achievements if the development of an abnormal gait is to be as far as possible averted.

As children with early signs develop, further signs characteristic of spasticity appear. The child's limbs resist stretching only when force is sharply applied to counter the postural tendency, and this resistance yields to sustained traction. Force meets no resistance where it assists the return of the limb to its habitual posture. Then, the two signs already noted in the infant become more marked, and with the older child lying in the supine position, when the ankles are firmly grasped, the thighs rotated inward and sharply abducted, the hips and knees flex abruptly and the lumbar curve increases as resistance occurs (Fig. 2). With the child in the prone position with the legs extended, the pelvis held firmly down and the hip adducted, when one leg is passively flexed at the knee, the hip on the same side flexes; at this stage no plantar stimulus is required to evoke the reaction. With the child placed and supported on his side with his legs extended in line with the trunk, when the uppermost leg is passively drawn backward and abducted, the under leg rises 


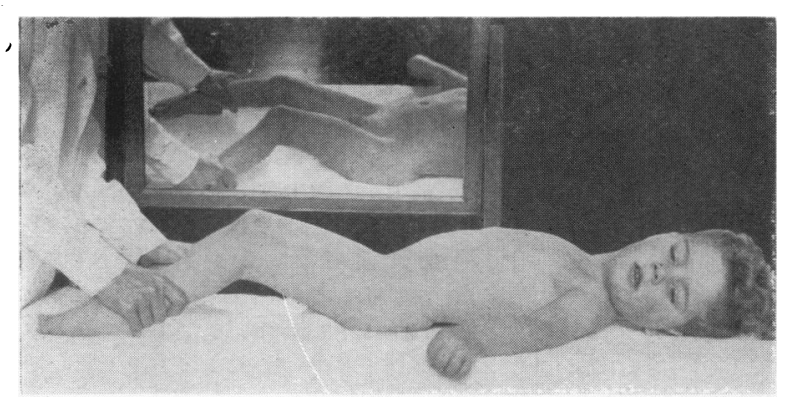

FIG. 1.

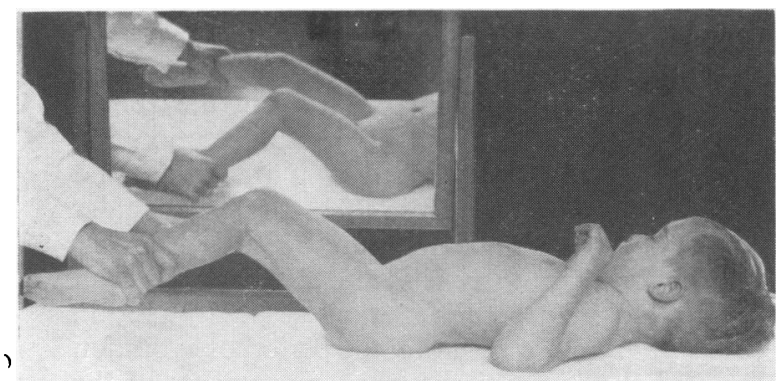

FIG. 2.

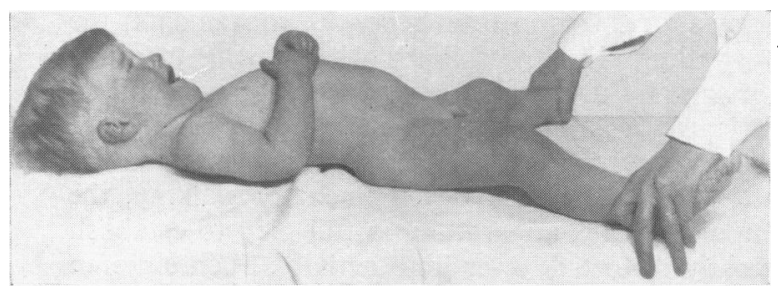

FIG. 3.

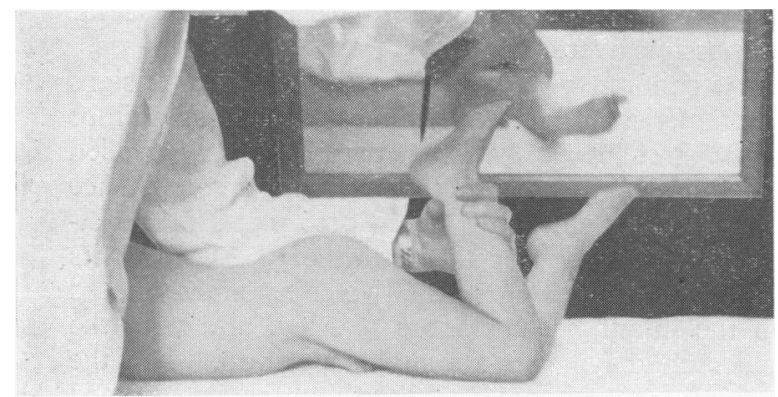

FIG. 4.

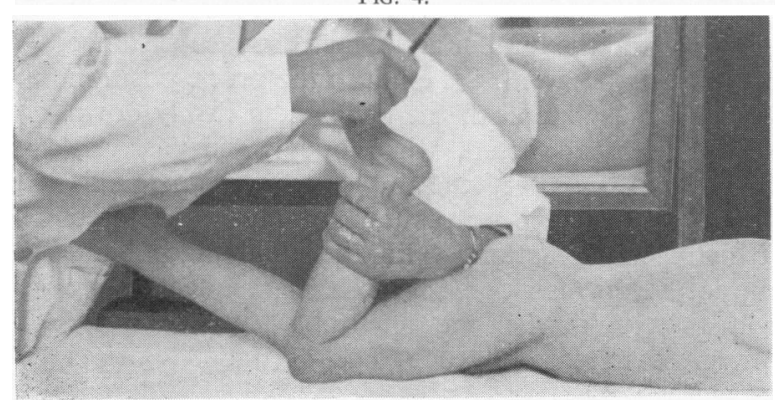

FIG. 5.

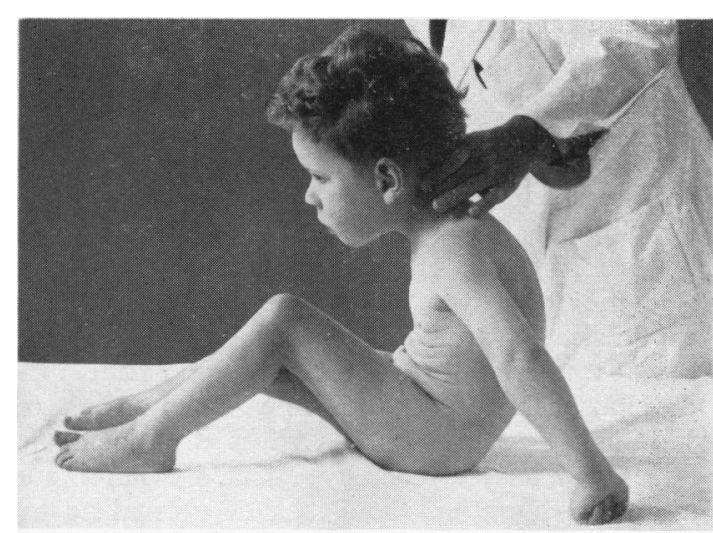

FIG. 6.

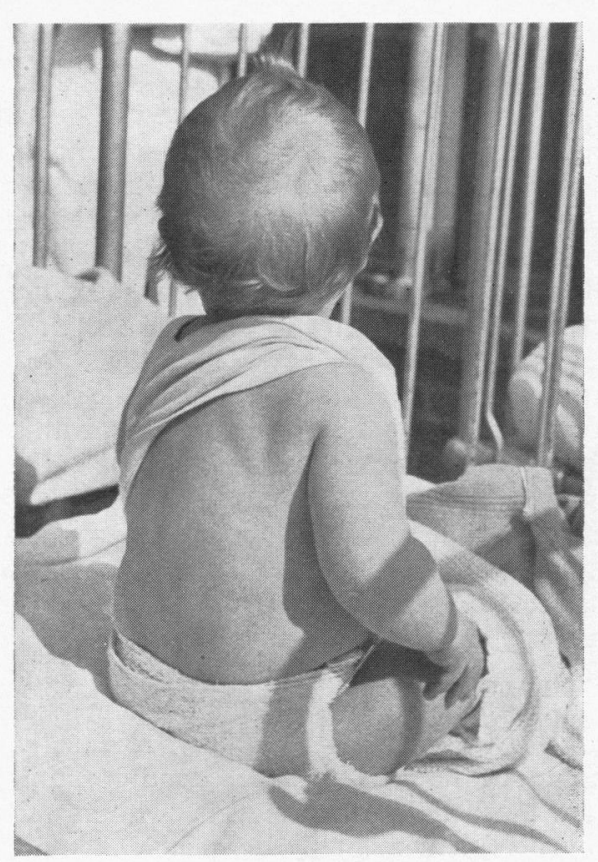

FIG. 6a.

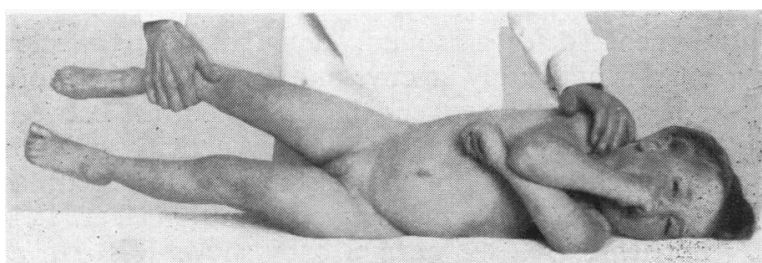

FIG. 7. 
In Fig. 1, with the child (aged 3, spasticity not fully developed) supine, the ankles are grasped, the thighs rotated inward and sharply abducted, the hips and knees flex abruptly. Fig. 2 shows an older child (aged 5) in whom the sign is more marked. Fig. 3 shows the reaction of a normal child for comparison.

In Fig. 4, when with the child prone and the pelvis held in contact with the table, one of his knees is passively bent and the sole of that foot stroked firmly, the hip on the same side flexes. Fig. 5 shows a similar reaction of the other hip (same child as Figs. 1 and 6).

Fig. 6 shows same child as in Figs. 1, 4 and 5 pushed into a sitting position and Fig. 6 a a normal child for comparison.

Fig. 7 shows same child as in Fig. 2 supported on his side with the legs extended in a line with the trunk; when the uppermost leg is passively drawn backward and abducted the under leg rises off the table.

Fig. 8 shows a normal child suspended by one ankle. This child is the twin sister of the child with spasticity shown in Figs. 8a-8d. Figs. 8a and 8b show retained extension of the freed leg on release of either ankle.

Fig. 8c shows the freed leg pushed into flexion. Fig. 8d shows the return of the freed leg to extension when pressure is released. Compare the postures of the head and arms with those of the normal twin child.

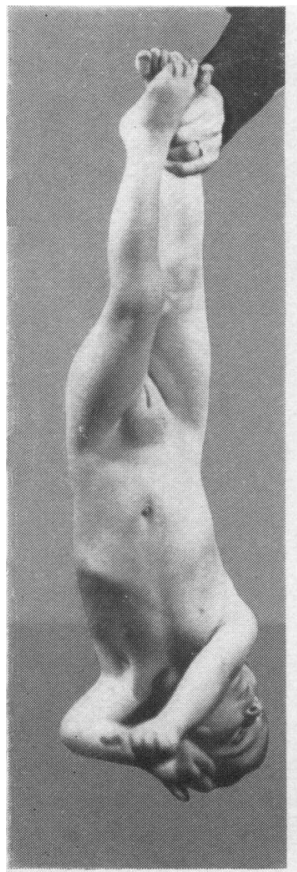

FIG. 8a.

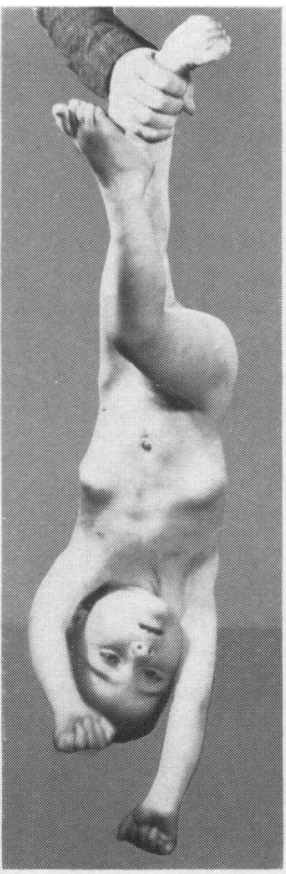

FIG. 8 b.

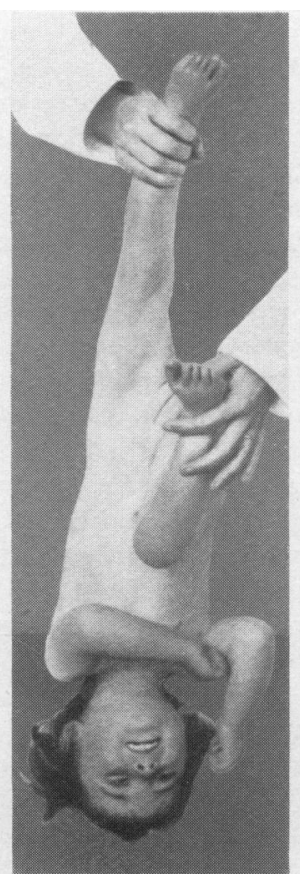

FIG. 8c.

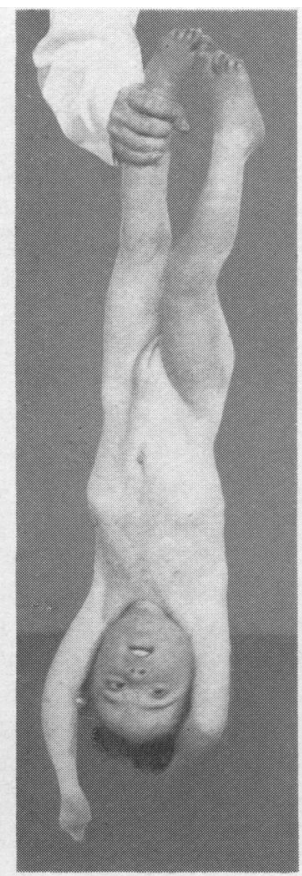

FIG. 8d.

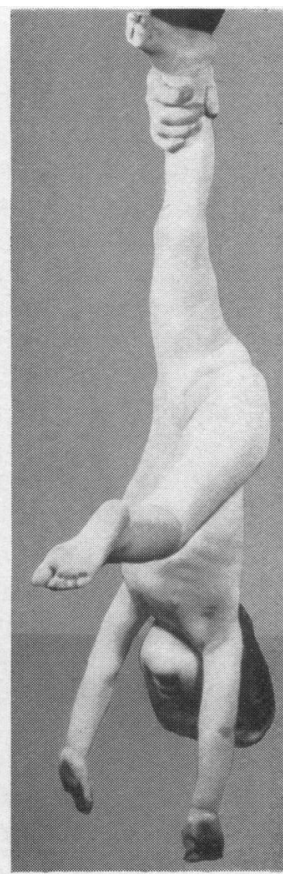

FIG. 8. 
off the surface on which it was placed (Fig. 7). With the child suspended by the ankles, the feet show inversion and plantar flexion (in contrast to the normal straight, strongly dorsiflexed feet), and on release of one ankle the freed leg may remain extended (Figs. $8 a$ and $8 b$ ) or if the leg flexes the thigh rotates in; if the leg remains extended, when the examiner pushes it into flexion and then releases pressure from the sole of the foot, the leg spontaneously returns with characteristic precision to its former extended position (Figs. 8c and 8d). When the child is pushed from a supine into a sitting position with the examiner's hand behind his neck, his head may fall forward or 'poke', the back is rounded as in a young infant or is noticeably convex backward in the lumbar region; the legs show internal rotation at the thigh, flexion at the knee, plantar flexion at the ankle; the child's weight rests on the sacrum instead of on the tuber ischii (Fig. 6).

By the time these signs are present, whatever other abnormalities are found, a diagnosis of generalized spasticity could generally be made on better known pyramidal signs. The tendon jerks frequently show briskness. 'Spastic' briskness, moreover, is characteristic, the response being identical on repetitive stimulation, each response being of sharply limited range. This is usually particularly well seen at the knee joints. Clonus which is abnormally sustained may frequently be easily elicited at the ankle joints, especially with the child in the prone position with the knee kept passively flexed during application of the stimulus. The great toe may extend in a deliberate manner when the sole of the foot is firmly stroked. In addition to these well-known signs in these cases, with the child lying supported on his side with the legs extended, when the uppermost leg is passively drawn backward, abducted, and then released, it falls and remains with the hip flexed on the surface on which the child is lying. This sign is invariably present in hypotonic infants who later develop spasticity. In children with gross spasticity in whom this sign persists dislocation of the hips may be present or may supervene. Attitudes of affected children are determined by the character of their physical activity which includes the voluntary as well as the reflex aspect of movement. Abnormally sustained attitudes constitute deformity and this develops in most cases because voluntary movement is slowed and limited by the motor defect.

In the absence of hearing deficiency clear speech develops in intelligent children with spasticity; on the other hand, where there is mental defect, development of speech may be retarded and this abnormality is often mistaken for dysarthria, especially where other signs of retardation include imperfect mastication and dribbling; in such cases the palatal reflex is unimpaired, as it is in mentally abnormal children with spasticity whose speech is clear but 'chattering'. In contrast, in other types of motor disorder the palatal reflex is usually defective and there is dysarthria in addition to dribbling and imperfect mastication. Among signs of mental abnormality, squinting is common in children with spasticity, but this defect seldom persists throughout childhood and should be distinguished from persistent strabismus of other origins.

\section{Rigidity}

The other type of neuropathological stiffening we find where signs of spasticity are absent, and this we call rigidity. We have never found this condition apart from signs of mental defect which include incoherence of movement often so gross as to be termed by many 'athetotic movements'. A history of rhesus incompatibility or of asphyxia pallida is obtained in these cases; in some there is a history of prolonged jaundice in infancy. If a young baby is very stiff and shows opisthotonus and other signs of widespread cerebral defect, a diagnosis of motor abnormality is easily made in addition to that of mental defect. If, however, stiffening is slight and not constant and infantile hypotonus is prominent a diagnosis of motor deficiency may be more difficult in the early stages. Yet, in all cases of rigidity signs of both motor and mental abnormality develop as age advances, and when signs of mental activity are separated out from the physical activity of these children, neuropathological stiffness remains which differs from that of spasticity. In most affected infants the eyes tend to roll upward and the babies often appear to be blind because they do not focus; blindness is an uncommon complication, however. When these babies are brought into a sitting position the eyes no longer roll upward. To induce a sitting position in an infant who shows marked stiffness it is necessary to flex his head sharply, when the child's body flexes as it does in the Landau reflex. Many infants with pathological rigidity are found to be partly deaf, but deafness is often overlooked where the mental defect is gross. Abnormal responses to skin stimulation are often found.

In children with rigidity even where there is severe motor and mental defect, motor characteristics change somewhat with increasing age. Where stiffness was constant it becomes intermittent, while those babies who were only slightly stiff develop greater rigidity which also is intermittent. Thus, the clinical features which develop are identical in cases which show rigidity, though the degree of abnormality varies from child to child. The combination 
of stiffness and gross incoherence of movement which develops in most affected children is the product of both motor and mental processes which are impaired in varying degree. When signs of mental activity are subtracted from physical activity the rigidity which remains is the diagnostic feature as far as motor abnormality is concerned. Children with rigidity of neuropathological origin showing intermittent stiffness are those whom we describe as having variable rigidity, and in these resistance to passive movement of a limb may abruptly cease and the limb become limp. In contrast to the resistance of spasticity that of rigidity is not altered by altering the speed of the passive movement. Stiffness and limpness of the child's limbs and body alternate abruptly. The alterations are not like those of 'cog-wheel' rigidity but are complete changes in the pliability of the limb and are usually associated with alterations of attitude of other parts of the body. Simultaneous changes of pliability are found in different limbs. This type of neuropathologically determined behaviour of the parts of the body seems to be unresponsive to the child's will; movements are usually stiffly performed with the limbs and body contorted, and incoherence of these movements may be very marked. Attitudes of head, limbs, and body, that is, postures characteristic of the mental and motor activity remaining to affected children, usually become abnormally sustained. For example, the arms may be rigidly extended with internal rotation at the shoulder, sometimes so extreme that the back of the elbow joint is turned toward the front of the body; the legs may similarly be rigidly extended and internally rotated at the hip joint. Even where these attitudes are so persistent as to constitute deformity and are even maintained during sleep, they may yield suddenly under the examiner's hands. In addition to these signs, the palatal reflex is abnormal. Rarely this is found to be hyperactive, in most cases it is almost inactive, dribbling of saliva is usually present, and swallowing, chewing and speech are deficient. Abnormality of respiratory movements is frequently marked throughout early childhood, and digestive troubles, including flatulence and vomiting, are common. Affected children are often ill nourished, and, as they grow older, deformity of the chest becomes marked in many cases. Sustained clonus, especially at the ankle joints, is often present, though the tendon jerks, when obtained, are normal, and, after infancy, the plantar response is equivocal or flexor. Tendon jerks and a clear plantar response are difficult to obtain in the presence of sustained rigidity.

Children with variable rigidity and incoherent movement must be distinguished from those in whom incoordination is of motor origin only and the stiffness which develops with age is the result of unimpaired mental processes activating impaired motor processes, and they must also be distinguished from children with spasticity. In children with variable rigidity the following abnormal reactions are found. When, with the child supine, the ankles are firmly grasped, the thighs rotated in and abducted, resistance may be encountered; in contrast to that of spasticity, such resistance is not augmented by increased speed of passive abduction, and instead of 'melting' under sustained traction it may abruptly yield at any phase of the passive movement or be sustained throughout. Attempts to flex the extended arm or leg may meet with similar resistance. Often there is no resistance to an attempt to extend the same limb immediately afterwards. When, with the child placed prone, with his pelvis held in contact with the surface on which he is lying, one of his legs is passively flexed at the knee and the sole of that foot firmly stroked, a withdrawal reaction takes place. This is characteristic of 'retardation' and occurs in a cumulative series of jerks as the stimulus is repeated; it thus differs from the reaction found in the presence of spasticity. With the child placed and supported on his side with his legs extended, the uppermost leg momentarily remains in abduction when lifted and released unless this manoeuvre is performed during a phase when resistance abruptly wanes. With the child suspended by the ankles, the feet show plantar flexion, and on release of one ankle the leg may remain stiffly extended, though the knee is not usually turned in as it is in the child with spasticity; when pushed into flexion the leg usually remains in the flexed position. When the child is pushed from a supine into a sitting position with the examiner's hand behind his neck, the trunk may fall forward with the head flexed and the back rounded. If this reaction is not immediate, passive flexion at the neck will usually produce it; the legs may be widely abducted and extended or may show flexion with the feet plantar flexed. In affected children mental development is slow and limited and symmetrical attitudes of the limbs only gradually succeed the tonic neck reflex attitude described by many authors for early infancy and for decerebrate rigidity. The physical characteristics of the older child, apart from the intractable fluctuating rigidity from which we have named this condition, depend largely on his mental capacity and hence are subject to modification by mental and emotional development.

Incoordination

Incoherence of mental origin in physical activity 
differs from incoordination of motor origin. We have been able to distinguish only two types of motor incoordination in children with infantile cerebral palsy. These we call respectively ataxia and athetosis. Incoordination of either type varies in degree from case to case, but the reactions characterizing each type remain constant. While ataxia may be present in children with a history of gross signs of cerebral abnormality in early infancy, athetosis is found only in children with a history of asphyxia livida neonatorum who showed no gross early signs, or, rarely, in those with a history of early normality and subsequent asphyxia during a paroxysm of whooping-cough.

Infantile ataxia is, in our experience, a relatively rare type of palsy and the clinical picture which develops conforms rather closely with that seen in adult ataxia. When signs of mental activity are subtracted from physical activity the motor defect is that there is too little stiffening. The child with ataxia thus finds himself unable to move effectively against even the ordinary opposition of gravity. In the supine and prone positions he lies with widely abducted, externally rotated and extended limbs. with his feet in eversion and plantar flexion, and shows no resistance to passive displacement. With the child placed and supported on his side, the uppermost limbs fall when lifted and released. The leg falls into flexion if the hip is flexed before the limb is released and extension if it is extended. With the child held suspended by the ankles, the head fails to extend; the feet show eversion, and on release of one ankle the free leg falls into abnormally wide abduction. Well recognized signs, such as nystagmus or other eye defects, slurring speech, absent knee jerks, 'pendulum' reactions of the limbs, may also be found. In some cases hearing is defective. Where there were gross early signs mental defect is shown as development proceeds.

The unsteadiness of movement which underlies developing voluntary activity of children falling into the remaining group of infantile cerebral palsies is usually described as athetosis. However, the term athetosis (without fixed position) is often used to describe any incoherence or incoordination of movement irrespective of its origin, and this is confusing because there is no other diagnostic term to distinguish this last group from those in which signs already described are found. For lack of a diagnostic term for these cases, we have restricted to them our use of the term athetosis. In them, the sole abnormal characteristic of physical activity is generalized unsteadiness of movement including that of speech, and this abnormality must be distinguished from the incoherence of movement associated with mental defect and from the hyperreflexia already described, as well as from the ataxic type of incoordination. Partial deafness is occasionally found in children with athetosis. Though affected infants show abnormality of breathing, sucking and swallowing as a sequel of blue asphyxia, and though hypotonus is a sequel of their motor insufficiency, since gross signs are absent, early diagnosis is rare. Yet, in these cases early diagnosis is particularly desirable as even where the motor defect is of severe degree, normality of mental processes is found and hence under appropriate management the prognosis is favourable. With mental development, however, and especially where there is also deafness, the bizarre expression enforced upon mental activity by the motor disorder may mislead the observer. We are accustomed ordinarily to deduce people's mental and emotional processes from the expression of these in physical activity-in attitudes, in speech, in facial play, in gesture and in achievements. We assess mental growth in children by alteration in their physical expression and, normally, this includes not only increase in physical attainments but also decrease in physical insecurity and incoordination. Where, however, the motor activity underlying developing expression lacks the normal attribute of steadiness, affected children inevitably shǫw insecurity and incoordination disproportionate to their mental growth. Hence, their physical instability may create a false impression of emotional instability or even of mental deficiency. Because ordinary achievements are too difficult for them such children grimace and stiffen with effort and when emotionally disturbed. These are normal reactions and it is normal to throw out the arms and plantar flex the feet when physically insecure, but in these children even these reactions are subject to distortion equally with other fragments of motor activity. Hence, when compared with normal easy movement, difficult movement and the effects of the difficulty may alike be taken for morbid signs and the results attributed to two coexistent motor lesions: a diagnosis of combined extrapyramidal and pyramidal abnormality is then given.

Stiffening of effort tends to 'fix' attitudes of insecurity and of effort, and, in time, these sustained attitudes constitute deformity. Further, as is well known, the tendon jerks may be affected by physiological stiffening, and incoordination may mask the nature of the plantar response; these irregularities may be mistaken for pyramidal signs. Since, however, the abnormal degree of stiffening exerted by these children is associated with effort it diminishes as effort is relaxed and tendon jerks are then found to be normal. Further, stiffening tends to mask 
motor incoordination associated with mental activity. The motor state may be difficult to assess unless the complex interaction of motor abnormality and its results are separated. Reactions associated with mental processes are not found in early infancy, during sleep or at other times when mental activity is quiescent, in these phases 'athetosis' is not seen. When signs of mental activity are subtracted from the physical activity of affected children the diagnostic characteristic of motor incoordination remains. In contrast to the incoordination of ataxia where there is inadequate stiffening, the incoordination of athetosis results from inefficacious steadying mechanisms.

With the child in the supine and prone positions no abnormal resistance or lack of resistance is found when the legs are passively abducted, though the child may stiffen with effort. With the child placed and supported on his side, when the uppermost leg is lifted, the lowermost leg remains on the supporting surface beneath it; when released the uppermost leg momentarily remains abducted and usually shows marked unsteadiness and some stiffness of effort may be present. With the child suspended by the ankles, the feet show absence of the normal strong dorsiflexion and remain plantar flexed; on release of one ankle the leg may fall or may remain extended. When it falls the fall is wavering; if the extended leg is pushed down it stays down. Other signs of motor insufficiency in these cases include deficiency of the palatal reflex, inability to frown, raise the eyebrows, close the eyes normally or smile normally. Squinting is a normal phenomenon of early infancy, but it does not persist in children with athetosis, though the fists may be held closed, the infantile grasping reflex is not found, nor are other signs of mental retardation present.

\section{Hemiplegia}

Epilepsy, which is sometimes associated with combined mental and generalized motor defects, may also occur in cases of infantile hemiplegia. This last condition is usually easily distinguished from generalized motor defect; early flaccidity of one side of the body is sometimes noticed by vigilant parents. The signs which develop on one side are not dissimilar from those seen in generalized infantile spasticity; involuntary irregular movements of the disordered limbs may be seen in epileptic children with hemiplegia. In some cases the whole affected side is smaller than the other from early infancy; in other cases a discrepancy between the two sides of the body is not noticed until a later stage of development and seems to be accounted for by the relative inactivity of the palsied side in the absence of effective treatment. In the prone position with both knees flexed the child's body curves to the affected side. In our experience signs of mental abnormality are usually found in these children and their speech is usually retarded.

\section{Conclusion}

In conclusion, I have, perforce, recounted only some of the details of physical activity of motordeficient children which, I think, help to differentiate their motor defects, and I have not described differing types of mental defect. Nor have I referred to likeness in details of physical activity. Yet, intact structures in incomplete brains have common function with similar structures in complete brains, and hence there are likenesses in physical activity to be described; these demand interpretation no less than do differences. I think that when both differences and likenesses are considered together reasonable explanations occur for some of the features of motor abnormality which are often regarded as puzzling. For instance, the apparent left-handedness of numbers of affected children, the relatively normal speech of most children with spasticity, the manner of development of characteristic deformity in each type of motor defect, the good use of the hands usually developed in children with spasticity and the poor use of the hands so commonly found in children with athetosis.

\section{Summary}

From observation of developing physical activity in babies and young children the clinician deduces the normality or abnormality of their maturing brains.

In physical activity, however, related motor and mental functions of the brain are simultaneously represented and each is of considerable complexity. Hence, deficiency in either must be separately assessed and remaining function analysed, for on the nature of this depend the planning and the outcome of appropriate management.

Deficiencies of cerebral motor function are discussed in this paper. An account of some of the simpler details of physical activity from which the type of defect may be deduced is given. Signs of mental defect are not described here.

Differences distinguishing the four types of abnormal motor activity covered by the general term the infantile cerebral palsies are described; two of these four types are characterized by abnormal stiffening and two by incoordination. The two types of stiffness and the two types of incoordination are 
each separately described and differentiated. Likenesses in physical activity of affected children are not described. Relationship has been found between types and early histories.

Establishment of effective utilization of a deficient motor apparatus depends not only on the affected child's own efforts but also on the kind of help he receives in infancy. Thus rational physical handling can help all affected children apart from those in whom there is gross mental deficit. In a small proportion-perhaps $20 \%$ or even less-of all cerebral palsied children mental processes are entirely normal even where the motor disorder is extreme, and in these the prognosis is excellent if early appropriate help is given. For these especially should be made available the inestimable advantages of differential diagnosis and appropriate care during the stages of maximal plasticity of central nervous function.

I would like to thank Dr. David Lawson, the Physician Superintendent of Queen Mary's Hospital for Children, for permission to publish the photographs.

\section{REFERENCE}

Collis, E. (1953). Lancet, 1, 416. 